\title{
Inhalt des 1. Jahrganges
}

H. Bueß, Dr. med. et phil. Andreas Ruinella (ca. 1555-1620?), ein wenig bekannter Bündner Humanist, über die Zurückhaltung der Menses

R. von Fellenberg, Eine Vorlesung über den Kaiserschnitt aus dem Anfang des 19. Jahrhunderts

H. E. Fierz-David, August von Kekulés chemische Visionen

H.Fischer, Schweizerische Bibliographie der Geschichte der Naturwissenschaften und der Medizin 1938-1943 I.

- - Schweiz. Bibliographie der Geschichte der Naturwissenschaften und der Medizin 1938-1943 II.

- - Bitte des Herausgebers an die Mitglieder

- - Zur Erinnerung an Antoine Laurent Lavoisier (1743-1794)

- - Jean Strohl

- - Erinnerung an Vesal (1514-1564)

J.W.Goethe, Aphorismen zur Wissenschaftsgeschichte 36

G.Goldschmidt, Ein Pseudo-Apuleiusfragment in einer Zürcher Handschrift

E. Hintzsche, Ein neuer Brief von Alfonso Corti (1822-1876) 137

E. Olivier, Souvenirs sur A. C. Klebs

- - Un Régime pour garder santé donné au duc de Savoie par un gentilhomme vaudois

G. Piotet, Le Docteur Arnold-C. Klebs

G. Senn, Der Rebbau im antiken Griechenland nach Theophrast, Causae plantarum Kap. 11-16

J. Strohl und H. Fischer, Einführung

$R$. Wavre, Galilée et le problème du temps

E. Wölfflin, Einiges aus der Praxis von Galen

\section{Buchbesprechungen:}

J. Ackeret, Untersuchung einer nach den Euler'schen Vorschlägen (1754) gebauten Wasserturbine (Ed. Fueter)

Leonhardi Euleri, Opera ser. I. vol. IV. (Ed. Fueter)

Knut Hagberg, Carl Linnaeus (G. Senn)

E. Hintzsche, Alfonso Corti (1822-1876) (H. Fischer)

Sten Lindroth, Paracelsismen i Sverige till 1600. (G. Goldschmidt)

C. St. Smith and Martha Teach Gnudi, The Pirotechnia of Vanoccio Birincuccio (H. Fischer)

F. Stüßi, 200 Jahre Euler'sche Knickformel (Ed. Fueter)

M. Trembley, Correspondance inédite entre Réaumur et Abraham Trembley 